\title{
Depresión y ansiedad en los tiempos de la pandemia de COVID-19
}

\author{
Depression and anxiety during COVID-19 pandemic
}

\section{Humberto Nicolini*}

Laboratorio de Enfermedades Psiquiátricas, Neurodegenerativas y Adicciones, Instituto Nacional de Medicina Genómica, Ciudad de México, México

\section{Introducción}

La pandemia que venimos sufriendo desde este año 2020, a causa de la enfermedad por coronavirus SARS-CoV-2 (COVID-19), ha marcado nuestras vidas y ha modificado nuestras conductas, nuestras percepciones y el entorno.

Esta situación de salud ha obligado a los gobiernos a tomar medidas importantes de restricción de la actividad normal de la sociedad, tanto en nuestro país como en todo el mundo, con indudables beneficios para la salud pública, pero con secuelas importantes en la economía y la salud mental de la población.

Al presentar su informe de actuación sobre los efectos de la COVID-19 y la salud mental, el Secretario General de las Naciones Unidas, Antonio Guterres, destacó que las personas que corren mayor riesgo en la actualidad son los trabajadores sanitarios que están en la primera línea de atención, las personas mayores, los adolescentes y los jóvenes, las personas con afecciones de salud mental preexistentes y las que están atrapadas en conflictos y crisis. La Directora del Departamento de Salud Mental y Consumo de Sustancias de la Organización Mundial de la Salud, Déborah Kestel, dijo que se ha incrementado el número de personas con problemas de salud mental y que han aumentado las tasas de suicidio. Así mismo, el Director de la Organización Mundial de la Salud, el Dr. Tedros Adhanom Ghebreyesus, señaló que el impacto de la pandemia sobre la salud mental de la población es muy preocupante. El aislamiento social, el miedo al contagio y la pérdida de seres queridos se han agudizado debido a la falta de ingresos o del trabajo. Todo ello puede generar un escenario catastrófico para la salud mental. Sin embargo, cabe destacar que hasta la fecha no hay muchas publicaciones que investiguen el tema de la salud mental, tanto en México como en otros países. Existen pocos artículos observacionales en la literatura y muchos son de opinión o puntos de vista ${ }^{1,2}$.

\section{Problemas de salud mental en la pandemia de COVID-19}

De la literatura existente, los estudios han señalado que se han aumentado las cifras de diagnóstico de ansiedad a raíz de la pandemia de COVID-19. El diagnóstico de ansiedad frecuentemente se presenta con problemas de sueño y comorbilidad con depresión. Identificar las poblaciones con mayor riesgo de resultados adversos para la salud mental, incluidos los pacientes con COVID-19 y sus familias, individuos con morbilidad física o psiquiátrica existente, y trabajadores de la salud, es una tarea muy importante de la salud pública durante y después de la pandemia.

Otra línea de evidencia es la experiencia recabada de los afectados por el brote de Ébola de 2014-2016, que sugiere que la exposición a una pandemia puede estar asociada con síntomas de ansiedad, depresión y trastorno de estrés postraumático ${ }^{3}$.

\section{Correspondencia:}

*Humberto Nicolini

Periferico Sur 4809 
La ansiedad por la salud, que surge de la mala interpretación de las sensaciones corporales y los cambios percibidos, puede tener un efecto protector en la vida cotidiana. Sin embargo, durante un brote de una enfermedad infecciosa, en particular si la información es cambiante o exagerada en los medios de comunicación, la ansiedad por la salud puede volverse excesiva y llegar a lo que llamamos hipocondría. A nivel individual, esto puede manifestarse como comportamientos desadaptativos (consultas médicas repetidas, evitando la atención médica incluso si se está realmente enfermo, atesorando utensilios de limpieza particulares como papel de baño, sanitizantes, etc.), y a un nivel social más amplio puede generar desconfianza en las autoridades públicas y sentimientos de ser el chivo expiatorio de poblaciones o grupos particulares.

Es importante prestar la atención adecuada a las condiciones psiquiátricas específicas que pueden ser iniciadas o exacerbadas por el desastre. Los centros de atención de salud mental para enfermos graves, los centros de medio camino, las consultas externas, las juntas de pacientes con adicciones y las consultas externas psiquiátricas han sido suspendidos temporalmente. Esto ha sido el detonante que ha disparado la descompensación de una gran cantidad de pacientes con trastornos mentales que venían llevando un control adecuado. Esta experiencia ha sido global. Por otra parte, sabemos que las personas que viven en la calle son una población muy vulnerable, ya que una proporción significativa de ellos padecen trastornos mentales o problemas adictivos graves, y se ha visto que es una población muy susceptible a la COVID-19.

Los individuos dentro del espectro autista, así como aquellos con patologías psiquiátricas como la depresión crónica y la esquizofrenia, tienen mayor probabilidad de padecer enfermedades crónicas, comorbilidad autoinmunitaria y consumir más fármacos crónicamente, lo cual puede aumentar la vulnerabilidad al COVID-19.

Por otro lado, debido a la patología psiquiátrica de base, muchos pacientes experimentan soledad y aislamiento, aunado a la restricción del acceso a los sitios habituales de terapia o al personal de apoyo que los atiende, lo que seguramente está causando una mayor descompensación en las patologías existentes. Aunque muchos de estos servicios no son considerados esenciales, para este tipo de pacientes la ayuda asistencial es fundamental para su higiene personal, sus patrones de alimentación o la toma de medicamentos. Además, se deben tomar en cuenta los graves problemas económicos de la población psiquiátrica, en la que es habitual que haya altas tasas de desempleo.

El aumento del consumo del alcohol y drogas es otra área de preocupación importante en el campo de la salud mental. Hay diferentes reportes que han señalado que esto ha ocurrido debido a la situación del confinamiento y el estrés por la pandemia.

En China se ha reportado un incremento de los síntomas depresivos en la población juvenil del $22.6 \%$, que es mayor que lo reportado en otras investigaciones en escuelas primarias (17.2\%). Durante la pandemia de COVID-19, la reducción de las actividades al aire libre y de la interacción social puede haberse asociado con un aumento de los síntomas depresivos en los niños. También se encontró que el $18.9 \%$ de los estudiantes tenían síntomas de ansiedad, una prevalencia mayor que en otras encuestas. Estos hallazgos sugieren que las enfermedades infecciosas graves pueden influir en la salud mental de los niños como lo hacen otras experiencias traumáticas ${ }^{4}$.

Quizás ningún grupo de personas con enfermedades mentales se ve tan directamente afectado por el brote de COVID-19 como aquellas que viven con el trastorno obsesivo compulsivo (TOC). Paradójicamente, son «expertos por experiencia» en el intento de evitar peligros mediante la actuación con comportamientos compulsivos. En particular, el aumento de la ansiedad por el virus está alimentando los temores obsesivos existentes de contaminación en algunas personas con TOC y desencadenando aún más acciones compulsivas dañinas. Para estas personas, el coronavirus puede convertirse en todo lo que piensan. De hecho, algunos pacientes con TOC relacionado con la contaminación están expresando dudas sobre la racionalidad de las terapias que han estado aplicando. Varios pacientes han dicho a sus médicos que «tenían razón todo el tiempo", ya que ahora todos se parecen a ellos. Un grupo de trabajo de expertos clínicos del International College of Obsessive Compulsive Spectrum Disorders y la Obsessive-Compulsive and Related Disorders Research Network del European College of Neuropsychopharmacology han realizado una declaración consensuada con el objetivo de brindar orientación pragmática lo antes posible a los médicos para manejar este complejo desafío ${ }^{1}$. Dentro de estas recomendaciones, que aplican para cualquier patología psiquiátrica, se indica que en primer lugar hay que tener un abordaje compasivo, entendiendo el contexto sociocultural del paciente; en segundo lugar, debe hacerse una buena historia clínica; en tercer lugar, 
evaluar el riesgo de suicidio; en cuarto lugar, evaluar el uso de Internet y el consumo de noticias; y finalmente, proveer adecuada psicoeducación acerca de los riesgos físicos y emocionales de la COVID-19.

Si hay un problema psiquiátrico, hay que revisar el tratamiento farmacológico y adecuar la continuación de los programas de psicoterapia (telepsicoterapia); los pacientes en tratamiento con estimulación magnética transcraneal o con estimulación cerebral profunda deben pausarla hasta el fin del confinamiento. Hay que asegurar actividades ocupacionales, rutinas y ritmos circadianos, ya que el aislamiento no necesariamente es soledad. También es importante señalar que en el tratamiento psiquiátrico de los pacientes con COVID-19 hay que tener cuidado con las benzodiacepinas por sus efectos sobre el sistema respiratorio. Algunos retrovirales aumentan el intervalo QT del electrocardiograma, lo cual puede interactuar con los medicamentos antipsicóticos y anticonvulsivos.

\section{Violencia familiar}

El incremento de la violencia dentro de las familias es un relato común en esta pandemia. Las medidas para quedarse en casa conllevan un mayor riesgo de que los niños sean testigos o sufran violencia y abuso. Los niños con discapacidades, los niños en entornos abarrotados y los que viven y trabajan en las calles son particularmente vulnerables. Otros grupos que corren un riesgo particular son las mujeres (en especial aquellas que hacen malabares con la educación en el hogar, trabajan desde el hogar y realizan las tareas domésticas), las personas mayores y las personas con afecciones de salud mental preexistentes. Varias publicaciones han identificado poblaciones que pueden ser más vulnerables al impacto en la salud mental durante y después de la pandemia de COVID-19, y algunas de ellas han proporcionado sugerencias sobre intervenciones y provisión de servicios. Los grupos vulnerables identificados por estos autores incluyen adultos mayores, trabajadores migrantes sin hogar, mujeres embarazadas con enfermedades mentales y estudiantes chinos que están en el extranjero ${ }^{5}$.

\section{Economía y salud mental}

Los efectos globales en la economía secundarios a la pandemia son incalculables. La inestabilidad del empleo, la reducción del ingreso en las familias y el aumento de las deudas son sin duda quejas crecientes que están afectando la salud mental de la población. Sin desestimar estos efectos, parecería también que en ocasiones nuestros cerebros se enfocan mucho más en lo malo, lo negativo y lo catastrófico. Es común que la crítica destructiva, la atención incesante a las malas noticias de muertes y desastres, o a las calamidades que ocurren a las personas, sean los titulares de periódicos y noticieros en lugar de los logros y éxitos de la humanidad. A este sesgo de percepción se le ha llamado «efecto de la negatividad». Se han hecho experimentos psicológicos en los que a los sujetos que participan se les proponen paradigmas, y cuando hay pérdidas del dinero que se les otorga en el experimento les afecta emocionalmente mucho más que cuando ganan la misma cantidad. Se ha tratado de ver si hay situaciones en las que los eventos «buenos» compensen o tengan un mayor impacto que tener el evento "malo", y no se han encontrado. El sesgo de negatividad de nuestro cerebro tiene sentido evolutivo porque mantuvo a nuestros antepasados alertas ante los peligros fatales, pero distorsiona nuestra perspectiva en el entorno mediático de hoy. El constante aluvión de malas noticias y de crisis nos hace sentir impotentes y nos deja innecesariamente temerosos y enojados. Sin embargo, una vez que reconocemos nuestro sesgo de negatividad, el cerebro racional puede superar el poder del mal cuando es dañino y emplear ese poder cuando es beneficioso. De hecho, en lugar de desesperarnos por lo que está mal en la vida y en el mundo, podemos ver cuánto nos está yendo bien y cómo podemos estar aún mejor. Una estrategia muy importante es el fortalecimiento del autocontrol: ser realistas al establecer metas, controlar su progreso y mantener la entereza cuando se falla (resiliencia). Esto ha sido crucial en el restablecimiento de la salud financiera, así como del bienestar emocional ${ }^{6}$.

\section{Problemas en los trabajadores de la salud}

Los trabajadores de la salud tienen un riesgo significativo de resultados adversos para la salud mental durante la pandemia de COVID-19. Los trabajadores de atención médica de primera línea, que enfrentan grandes cargas de trabajo, decisiones de vida 0 muerte, y riesgo de infección, se ven particularmente afectados. Las razones para esto incluyen largas horas de trabajo, riesgo de infección, escasez de equipos de protección, soledad, fatiga física y separación de las familias 7 . En España, hasta el 20\% del personal sanitario se ha infectado, y el estar fuera de servicio 
les ha generado gran sentimiento de culpa por no poder seguir ayudando, además de la ansiedad propia de cualquier paciente ante la COVID-19: miedo a contagiarse, miedo a contagiar a los familiares y las carencias de los problemas de salud para lidiar con la sobredemanda propia de la pandemia. Hay una disociación y una represión de la labor diaria con la vivencia afectiva, pero la cronicidad del trabajo hará que eventualmente explote la crisis emocional. Una alternativa que se ha propuesto es ofrecer los servicios de grupos, hacer un sentido de actividades de esparcimiento entre médicos y favorecer la petición de ayuda; aun siendo personal de la salud, no automedicarse. Hasta la fecha se desconoce cuál es la prevalencia de portadores asintomáticos de SARSCoV-2 entre los trabajadores de la salud en la Ciudad de México, en contactos de pacientes con COVID-19, así como su relevancia clínica y epidemiológica.

\section{El médico como paciente}

Varios estudios han mostrado una alta prevalencia de síntomas de trastorno de estrés postraumático, ansiedad, miedo, depresión y frustración en los profesionales de emergencias involucrados en la epidemia de SARS de 2002-2004. Los síntomas más comunes incluyeron pensamientos recurrentes e intrusivos sobre los eventos experimentados durante la atención de pacientes, dificultades para quedarse dormido, de memoria y de concentración, hipervigilancia e hiperexcitación, arrebatos de ira, pérdida de motivación para trabajar, desregulaciones del estado de ánimo, conductas evitativas hacia las actividades y lugares de trabajo, abuso de alcohol o drogas, entumecimiento, aislamiento y desapego psicológico ${ }^{8}$. Se ha informado de que estas manifestaciones clínicas persistieron en los profesionales de la salud muchos años después del brote. Sin embargo, no todos desarrollaron estos síntomas; al parecer, los factores predictores para los profesionales de la salud son el sexo femenino, el bajo nivel socioeconómico, los eventos traumáticos durante la vida y la psicopatología premórbida.

Por otro lado, es bien sabido que los médicos y en general los trabajadores de la salud no somos los mejores pacientes, con altas tasas de automedicación, cuestionamiento a los colegas que nos tratan y pobre apego a los tratamientos ${ }^{9}$. Estos factores hacen más vulnerables al personal de salud a padecer formas más graves de enfermedad, e incluso se ha traducido en tasas más elevadas de suicidio que en la población general ${ }^{10}$.

En un estudio realizado en China se evaluó la presencia de psicopatología en 1257 trabajadores de la salud expuestos a la COVID-19. Una proporción considerable de participantes informó de síntomas de depresión (50\%), ansiedad (45\%), insomnio (34\%) y angustia (72\%). Enfermeras, mujeres, trabajadores de atención médica de primera línea y quienes trabajaban en Wuhan mostraron una mayor gravedad en todas las mediciones de síntomas de salud mental que otros trabajadores de atención médica ${ }^{11}$. Los trabajadores de atención médica de primera línea involucrados en el diagnóstico directo, el tratamiento y la atención de pacientes con COVID-19 se asociaron con un mayor riesgo de síntomas de depresión y ansiedad, y de insomnio. La falta de conectividad social ha asociado claramente el síndrome de burn-out (saturación por fatiga) en los médicos ${ }^{12}$. La conexión social, en particular el trabajo en equipo, aborda las necesidades psicológicas de la amistad y la estima, y no es un lujo, sino una necesidad humana básica que se incorpora a nuestra biología.

Los médicos son científicos, y como científicos es su responsabilidad aplicar lo que se conoce de la literatura de neurociencia y gestión sobre cómo crear entornos de trabajo positivos y de apoyo que conduzcan a la participación profesional y la atención óptima de los pacientes.

\section{Estrategias de manejo}

El aumento del número de personas que necesitan atención a la salud mental o apoyo psicosocial se ha visto agravado por la interrupción de los servicios de salud física y mental en muchos países. Además de la conversión de centros de salud mental en centros de atención para personas con COVID-19, los sistemas de atención se han visto afectados por el personal de salud mental infectado con el virus y el cierre de los servicios presenciales. Los servicios comunitarios, como los grupos de autoayuda para la dependencia del alcohol y las drogas, en muchos países no han podido reunirse durante varios meses.

En la respuesta inmediata y continua, las pautas de consenso enfatizan la importancia de las intervenciones que mantienen la calma, construyen comunidad y alimentan la esperanza. Desde una perspectiva de salud mental pública, las pautas para responder al trauma masivo y al desastre enfatizan la importancia de centrarse en la resiliencia. 
Es fundamental que las personas que viven con problemas de salud mental tengan acceso continuo al tratamiento. Los cambios en los enfoques para la prestación de atención de salud mental y apoyo psicosocial están mostrando signos de éxito en algunos países. En España, para garantizar la continuidad de la atención, los formuladores de políticas locales identificaron la psiquiatría de emergencia como un servicio esencial para permitir que los trabajadores de salud mental continúen los servicios ambulatorios por teléfono. Equipos de Egipto, Kenia, Nepal, Malasia y Nueva Zelanda, e incluso en México, entre otros, han aumentado la capacidad de las líneas telefónicas de emergencia para que la atención a la salud mental llegue a las personas necesitadas.

Debe continuar el apoyo a las acciones comunitarias que fortalecen la cohesión social y reducen la soledad, en particular para los más vulnerables, como las personas mayores. Tal apoyo es requerido por el gobierno, las autoridades locales, el sector privado y el público en general, con iniciativas como la provisión de paquetes de alimentos, visitas periódicas por teléfono a personas que viven solas y organización de actividades en línea para la estimulación intelectual y cognitiva.

La nueva experiencia en rápida evolución en la práctica diaria ha ayudado a aliviar muchas de la objeciones anteriores contra la telemedicina. La idea de que la telemedicina solo se presta para entrevistas es falsa, y se ha visto que las evaluaciones psiquiátricas simples se pueden realizar de forma remota. Otra preocupación común es que la telemedicina está restringida en gran medida a pacientes jóvenes con altos niveles de educación, dejando a muchas otras poblaciones desatendidas. Sin embargo, durante esta crisis, la adopción de nuevas tecnologías, aunque no es sencilla, resulta incómoda o no está disponible para todos los pacientes y médicos, ha sido sorprendentemente rápida. Debemos reconocer que la sociedad global ha cambiado y ahora muchos adultos mayores están capacitados para usar teléfonos inteligentes o videoconferencias. Las organizaciones profesionales, como la American Psychiatric Association, han introducido nuevas pautas para las visitas de telemedicina y han abordado los problemas legales críticos y los estándares de atención. La rápida expansión de la telemedicina en la práctica diaria actual también presenta desafíos, y una preocupación es sobre la privacidad y la seguridad. No todas las herramientas disponibles para videoconferencias cumplen con los estándares internacionalmente aceptados para proteger la confidencialidad de cada participante. Esto se aplica tanto a los pacientes como a los profesionales médicos que prestan los nuevos servicios remotos. Por lo tanto, los médicos que consideren ofrecer servicios remotos a los pacientes deben tomar todas las medidas necesarias para salvaguardar la privacidad de los participantes.

Ya se han publicado estudios controlados que han comparado la eficacia de la terapia cognitiva conductual presencial contra la telepsicoterapia. En un ensay $^{13}$, la terapia cognitiva conductual suministrada por Internet no parecía ser inferior a la presencial para la ansiedad por la salud, al tiempo que incurría en costos sociales netos más bajos. El formato en línea tiene el potencial de aumentar el acceso al tratamiento basado en la evidencia para la ansiedad por la salud. Los pacientes y las familias ahora tienen una mejor comprensión de la necesidad de visitas remotas. Existen poderosas anécdotas de la atención prestada de forma remota, que incluyen problemas muy emocionales, como la entrega de malas noticias. Sorprendentemente, muchos pacientes prefieren recibir esas malas noticias en la seguridad de su propio hogar en vez de en el entorno clínico más impersonal. La ampliación y la reorganización de los servicios de salud mental que ahora se necesitan a escala mundial son una oportunidad para construir un sistema de salud mental que sea adecuado para el futuro.

Los departamentos de salud mental deberían poder asumir una posición de liderazgo en el manejo psicosocial de situaciones similares a desastres, y esto requiere la adquisición de nuevas habilidades, en especial de cómo informar correctamente a la población sobre el riesgo, capacitar y diseminar procedimientos preventivos y de manejo efectivos para desastres, apoyar al personal de salud y rescatistas, y acompañar a aquellos que sufren un duelo.

\section{Conflicto de intereses}

El autor declara que no tiene ningún conflicto de intereses.

\section{Bibliografía}

1. Fineberg NA, Van Ameringen M, Drummond L, Hollander E, Stein DJ, Geller D, et al. How to manage obsessive-compulsive disorder (OCD) under COVID-19: a clinician's guide from the International College of Obsessive Compulsive Spectrum Disorders (ICOCS) and the Obsessive-Compulsive and Related Disorders Research Network (OCRN) of the European College of Neuropsychopharmacology. Compr Psychiatry. 2020;100:152174.

2. Priego-Parra BA, Triana-Romero A, Pinto-Gálvez SM, Durán Ramos C, Salas-Nolasco O, Manriquez Reyes M, et al. Anxiety, depression, attitudes, and internet addiction during the initial phase of the 2019 coronavirus disease (COVID-19) epidemic: a cross-sectional study in México. medRxiv preprint. https://doi.org/10.1101/2020.05.10.20095844. 
3. Jalloh M, Li W, Bunnell R, Ethier KA, O'Leary A, Hageman KM, et al. Impact of Ebola experiences and risk perceptions on mental health in Sierra Leone, July 2015. BMJ Glob Health. 2018;3:e000471.

4. Xie X, Xue Q, Zhou Y, Zhu K, Liu Q, Zhang J, et al. Mental health status among children in home confinement during the coronavirus disease 2019 outbreak in Hubei province, China. JAMA Pediatr. 2020 Apr 24;e201619. doi: 10.1001/jamapediatrics.2020.1619. Online ahead of print.

5. Rajkumar RP. COVID-19 and mental health: a review of the existing literature. Asian J Psychiatr. 2020;52:102066.

6. André N, Audiffren M, Baumeister RF. An integrative model of effortful control. Front Syst Neurosci. 2019;13:79.

7. Polizzi C, Lynn SJ, Perry A. Stress and coping in the time of COVID-19: pathways to resilience and recovery. Clinical Neuropsychiatry. 2020;17:59-62.

8. Conversano C, Marchi L, Miniati M. Psychological distress among healthcare professionals involved in the Covid-19 emergency: vulnerability and resilience factors. Clinical Neuropsychiatry. 2020;17:94-6.
9. Jin J, Sklar GE, Min Sen Oh V, Chuen Li S. Factors affecting therapeutic compliance: a review from the patient's perspective. Ther Clin Risk Manag. 2008;4:269-86.

10. Reger MA, Stanley IH, Joiner TE. Suicide mortality and coronavirus disease 2019 - a perfect storm? JAMA Psychiatry. 2020 Apr 10. doi: 10.1001/jamapsychiatry.2020.1060. Online ahead of print.

11. Lai J, Ma S, Wang Y, Cai Z, Hu J, Wei N, et al. Factors associated with mental health outcomes among health care workers exposed to coronavirus disease 2019. JAMA Netw Open. 2020;3:e203976.

12. Southwick SM, Southwick FS. The loss of social connectedness as a major contributor to physician burnout. Applying organizational and teamwork principles for prevention and recovery. JAMA Psychiatry. 2020 Feb 19. doi: 10.1001/jamapsychiatry.2019.4800. Online ahead of print.

13. Axelsson E, Andersson E, Ljótsson B, Björkander D, Hedman-Lagerlöf M, Hedman-Lagerlöf E. Effect of internet vs face-to-face cognitive behavior therapy for health anxiety. A randomized noninferiority clinical trial. JAMA Psychiatry. 2020 May 13:e200940. doi: 10.1001/jamapsychiatry.2020.0940. Online ahead of print. 